\title{
Article \\ Modeling a Pre-Touch Reaction Distance around Socially Touchable Upper Body Parts of a Robot
}

\author{
Dario Alfonso Cuello Mejía ${ }^{1,2, *(0)}$, Hidenobu Sumioka ${ }^{1}\left(\mathbb{D}\right.$, Hiroshi Ishiguro $^{1,2}$ and Masahiro Shiomi $^{1}$ \\ 1 Hiroshi Ishiguro Laboratories and Interaction Science Laboratories, ATR, Kyoto 619-0237, Japan; \\ sumioka@atr.jp (H.S.); ishiguro@sys.es.osaka-u.ac.jp (H.I.); m-shiomi@atr.jp (M.S.) \\ 2 Intelligent Robotics Laboratory, Department of Systems Innovation, Graduate School of Engineer Science, \\ Osaka University, Osaka 565-0871, Japan \\ * Correspondence: cuello.dario@irl.sys.es.osaka-u.ac.jp; Tel.: +81-080-6629-4768
}

Citation: Cuello Mejía, D.A.;

Sumioka, H.; Ishiguro, H.; Shiomi, M. Modeling a Pre-Touch Reaction Distance around Socially Touchable Upper Body Parts of a Robot. Appl. Sci. 2021, 11, 7307. https://doi.org/ 10.3390/app11167307

Academic Editor: Subhas

Mukhopadhyay

Received: 19 July 2021

Accepted: 6 August 2021

Published: 9 August 2021

Publisher's Note: MDPI stays neutral with regard to jurisdictional claims in published maps and institutional affiliations.

Copyright: (c) 2021 by the authors. Licensee MDPI, Basel, Switzerland. This article is an open access article distributed under the terms and conditions of the Creative Commons Attribution (CC BY) license (https:// creativecommons.org/licenses/by/ $4.0 /)$.
Featured Application: Analyzing and modeling a pre-touch reaction in human-human interaction over upper body parts for implementation in human-robot interaction. A potential application would be the development of a robot's behavior in scenarios where touch interaction is needed, for example, social robots focused on medical applications.

\begin{abstract}
Although before-touch situations are essential to achieve natural touch interactions between people and robots, they receive less attention than after-touch situations. This study reports pretouch reaction distance analysis results around touchable upper body parts, i.e., shoulders, elbows, and hands, based on human-human pre-touch interaction. We also analyzed the effects of gender, approach side, speed, and acclimation in modeling the pre-touch reaction distance, and found that the distance around the hands is smaller than the distance around the shoulders and elbows, and speed and acclimation affect the distance. On the other hand, gender and approach side do not significantly affect the pre-touch reaction distance. Finally, we implemented the results in a male-looking android and confirmed that it reacted toward pre-touch based on the obtained model.
\end{abstract}

Keywords: social behaviors; touch interaction; human-human interaction; human-robot interaction

\section{Introduction}

Touch interaction with other people provides various positive effects [1-6], but, due to the COVID-19 pandemic, such interaction is physically and socially limited in the world. In this context, using social robots as a partner of touch interaction is a promising approach to cover the lack of human-human touch interaction since past studies reported several positive effects of such interaction [7-15]. Even if the effects of touch interaction with such social robots fail to fulfill the needs of interaction with actual people, people's negative situations can be mitigated.

As described above, many research works reported the effectiveness of touch effects in human-robot interactions (HRIs). However, these studies mainly focused on after-touch situations; dealing with pre-touch situations has received less focus. A few past research works focused on the pre-touch reaction distance for a robot [16] and a virtual agent [17], although they focused on faces. Therefore, knowledge about pre-touch interaction remains limited. Based on human science literature, a part of the upper body (i.e., the shoulders or arms, including elbow and hands) is mainly used in touch interaction with others [18]. Therefore, pre-touch interaction should consider such touchable upper body parts for natural touch interaction.

We regard pre-touch interaction research as an extension of proxemics in touch interaction contexts. People generally keep a certain distance from others, such as keeping a personal distance [19] in conversation interaction contexts, and such knowledge is well known. Many social robots refer to proxemics' knowledge to control their positions when 
interacting with people [20-33]. Inspired by the idea of proxemics, our ultimate goal is to establish the basis of proxemics in the context of pre-touch interaction as pre-touch proxemics. Such basic knowledge will be important for social robots that physically interact with people.

This study investigated the pre-touch reaction distance from typical touchable upper body parts. For this purpose, first, we conducted data collection to gather people's pretouch reaction data. Next, we conducted a statistical analysis of the collected data and implemented a pre-touch reaction model to our android robot as shown in Figure 1. Thus, this study aims to identify the minimum comfortable distance in human-human touch interaction around the upper body.

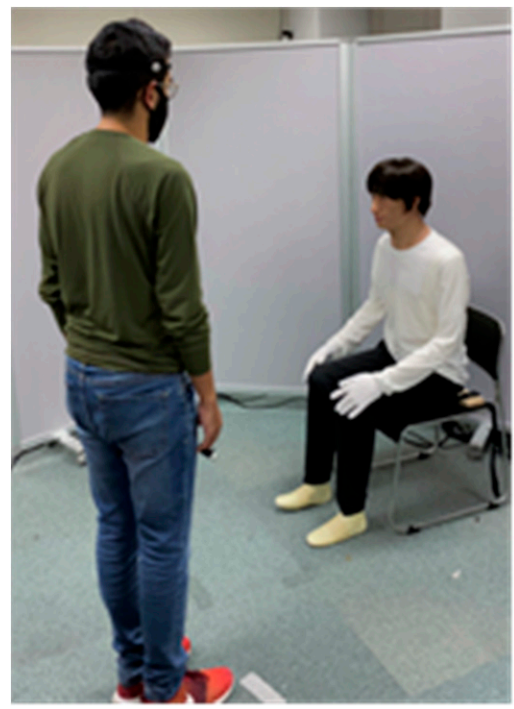

(a)

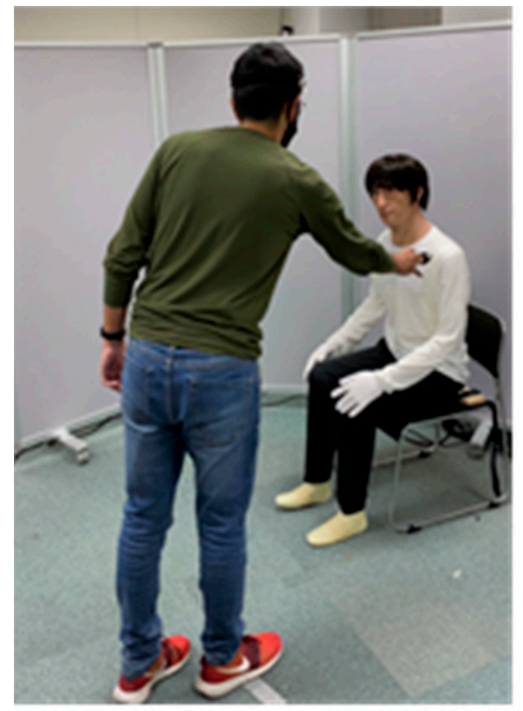

(b)

Figure 1. Android behavior to participant's approach: (a) initial position for starting touch interaction; (b) android looking at participant at a certain distance before being touched.

\section{Related Work}

\subsection{Touch in Human-Robot Interaction}

The main application of human-robot touch interaction is mental therapy for users. Several studies have investigated the merits of touch interaction with robots [7-10,34]. One famous therapy robot is a touchable seal robot (Paro) whose mental support effectiveness is well known worldwide [7]. Examples of commercially available, touchable pet-type robots include LOVOT (https: / /lovot.life/, accessed on 1 December 2020) and Qoobo (https:/ / qoobo.info/index/, accessed on 1 December 2020).

To achieve natural touch interaction between people and robots, robotics researchers have focused on communication cues, which have been broadly investigated in conversational settings [35-37]. However, such knowledge cannot be applied to touch interaction settings due to the differences in interaction settings or difficulties due to shorter distances. Therefore, robotics research on touch interaction is investigating appropriate communication cues under such settings $[14,38]$.

Robot appearances also influence touch interaction. For example, in human-human interaction, an acceptable body part for touching differs based on the relationship $[18,39]$. Which body part is touched also communicates a different meaning in the context of conveying emotions [40]. Researchers also investigated how a robot's body parts are essential to convey emotions by people/robots $[41,42]$. Other studies have focused on human touch interaction cues such as handshaking, describing its phases and analyzing both before-touch and after-touch actions [43], and defining methods for evaluating the human likeness of a robot handshake using a Turing-like test [44]. 
These studies described the importance of touch behavior in human-robot interaction from various perspectives. However, even though they provided rich knowledge about touch interaction, they focused on after-touch interaction situations, downplaying beforetouch situations. Unlike these studies, our study investigates pre-touch situations to understand people's perception of being touched. Therefore, we observed pre-touch proxemics data, modeled them, and implemented our model.

\subsection{Proxemics in Human-Robot Interaction}

When people interact with others, they appropriately adjust their position to others based on their relationships. This idea is known as proxemics [19]. Robotics researchers implemented this knowledge in social robots for smooth interaction with people in the context of navigation in daily environments [20-23], approaching people to start conversations [24], and displaying attitudes such as friendliness [25].

Researchers are also interested in the differences in the perceptions of proxemics between people and robots due to individual characteristics. Past studies have reported that people's personalities influence preferred distances with others [26-28]. Other studies have focused on such characteristics of robots as size [29,45], body postures $[30,31]$, and physical/virtual existence [46]. Other researchers have focused on mathematical functions to model the complex shape of personal spaces by considering the direction of the face and body $[47,48]$.

However, proxemics in touch interaction has received less focus. A couple of works have addressed pre-touch proxemics using a robot in a physical environment [16] and virtual agents in a virtual environment [17], although these studies concentrated on the face. Therefore, the knowledge of pre-touch proxemics is still limited. Compared to past proxemics studies in HRI, our study's unique point is that it deals with pre-touch proxemics around touchable upper body parts.

\section{Materials and Methods}

We analyzed human-human pre-touch interaction by data collection to obtain a pre-touch reaction distance for upper body parts.

\subsection{Data Collection}

\subsubsection{Overview}

Each data collection trial involved two participants who played both toucher and evaluator roles in order; the toucher makes physical contact with the evaluator, and the evaluator is touched by the toucher. The touch target parts are the shoulder, elbow, and the back of the hand, because previous studies investigated the pre-touch reaction distance around the face $[16,17]$ and reported that these body parts are typical acceptable body parts for touches [18]. Due to the sensitivity of touch interaction, we eliminated the lower body from the target body parts. The ethics committee at the Advanced Telecommunication Research Institute (ATR) approved this paper's methodology (20-501-4).

\subsubsection{Procedure}

Similar to past proxemics studies that modeled pre-touch $[16,17]$ and personal distances [23-28], we gathered self-reported preferred pre-touch reaction distance by the evaluators. Thus, the evaluator sat in a chair, and the toucher stood around $1.0 \mathrm{~m}$ in front of the evaluator, as shown in Figure 2. We fixed the evaluators' positions, including the face direction, and asked them to keep their gaze directed to the front. 


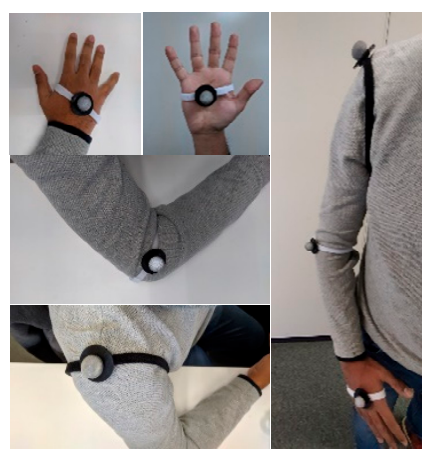

(a)

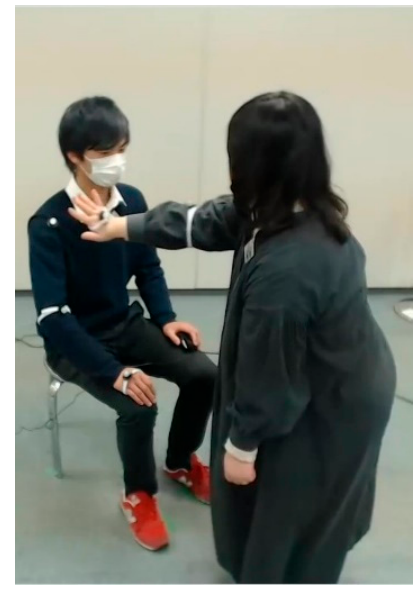

(b)

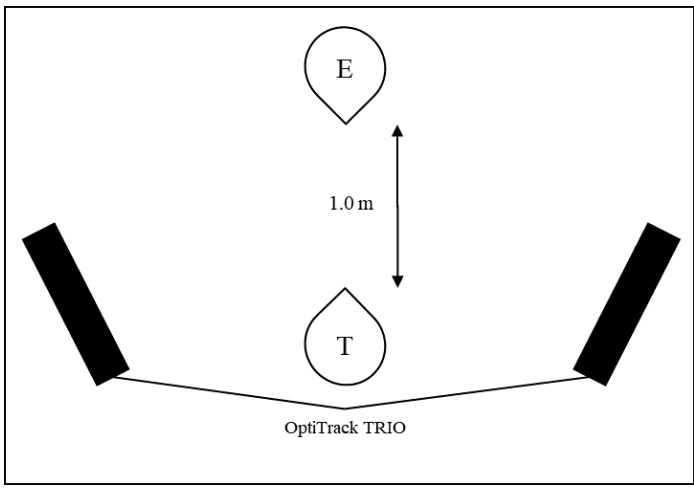

(c)

Figure 2. Data collection environment: (a) markers attached to both toucher and evaluator; (b) toucher is moving her hands toward evaluator's body; (c) data collection setup using two OptiTrack systems for tracking both.

The toucher slowly stretched out a hand toward the evaluator's body part; when the evaluator wanted the approaching hand to stop, he/she generated an audible signal by clicking a button. When the toucher heard the signal, he/she stopped immediately his/her hand, returned to the initial position, and continued the data collection. We asked the participants to move as naturally as possible and to use their dominant hands. Each trial's duration for each body part (shoulder, elbow, and hand) was two minutes.

The procedure for the evaluator's body parts was repeated for two cases. The first one was the front-right approach; the toucher approached from the right side of the evaluator. The second one was the front-left approach; the toucher approached from the left side. The order of the approaching sides (front-right or front-left) was counterbalanced between participants. Once the procedure was done, the participants changed roles and repeated the data collection.

\subsubsection{Recording Data}

In this data collection, we used two OptiTrack systems (Acuity Inc.) as a motion capture system to automatically track the positions of the body parts of both the touchers and the evaluators. We placed four markers for the former: shoulder, elbow, the palm of the hand (anterior), and the back of the hand (posterior). For the latter, we placed three markers: shoulder, elbow, and hand, as shown in Figure 2.

\subsubsection{Participants}

Sixteen pairs of Japanese participants (16 males and 16 females; eight male-female pairs, four male-male pairs, and four female-female pairs) joined our data collection. The participants met in the experiment for the first time, i.e., they were strangers. The average and S.D. of their ages was 36.39 and 11.63, respectively.

\subsubsection{Hypotheses and Predictions}

We hypothesized that the minimum comfortable distance is different due to the body part in the pre-touch proxemics modeling process around the upper body parts. Past studies reported that which body part is acceptable for being touched is different based on relationships $[18,39]$. Due to these studies, strangers were restricted to touching only the hands, regardless of cultural differences (East Asian and Western cultures), i.e., some might not welcome touches to their shoulders and elbows, and such hesitation would increase the minimum comfortable distance. Based on these considerations, we made the following hypothesis about the minimum comfortable distance. 
Prediction 1: The minimum comfortable distance around the hands is smaller than around the shoulders and elbows.

\section{Results and Implementation}

The data collection gathered 6593 bits of distance data. On average, the touchers' hands approached each evaluator's body part 34 times within two minutes. The total number of touching data was different between participants because of varied pre-touch reaction distances, approaching speeds, etc. Therefore, we used the average pre-touch reaction distance for each evaluator (i.e., 32) in the analysis instead of all the raw data. Figure 3 shows a part of the gathered data around each body part. Note that these positions are relatively transformed based on the 3D model body parts for visualization purposes. The obtained average pre-touch reaction distance for all the participants was $23.46 \mathrm{~cm}$, and the S.E. was 2.1.

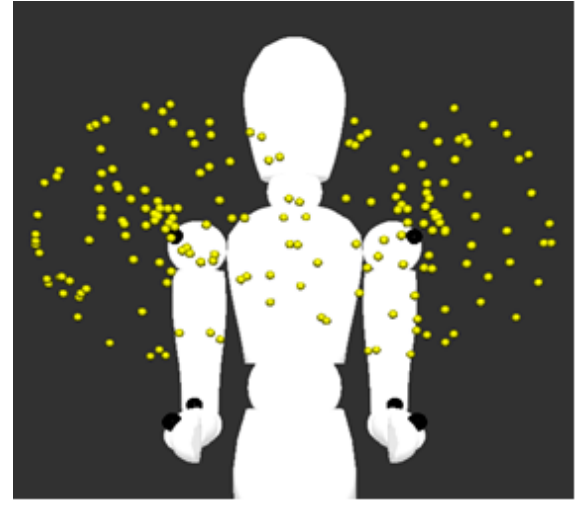

(a)

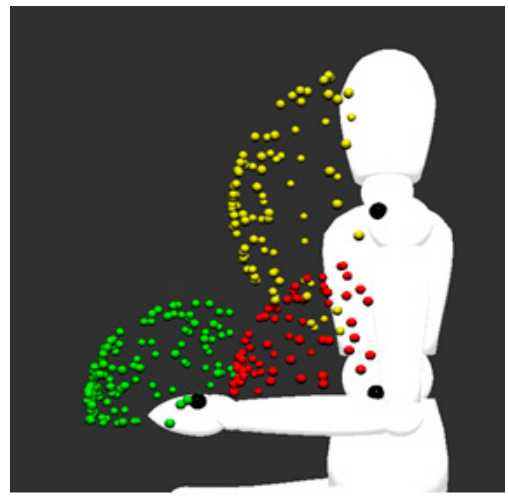

(d)

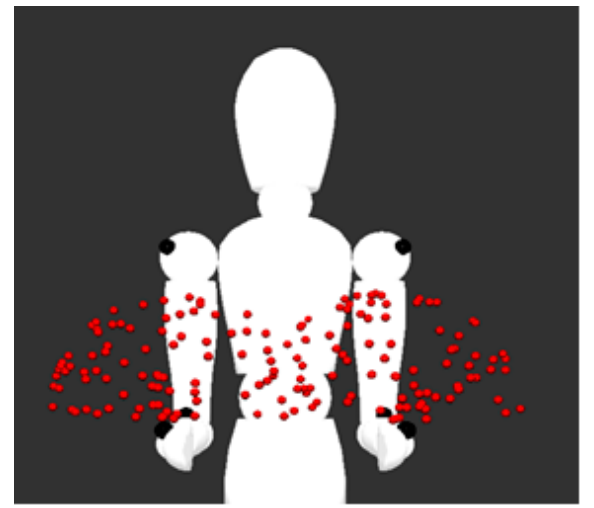

(b)

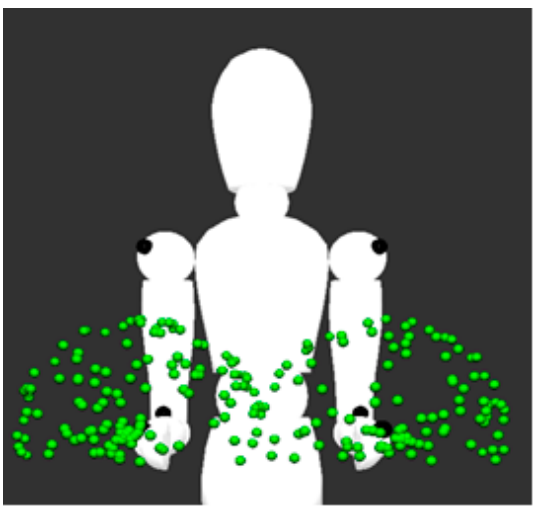

(c)

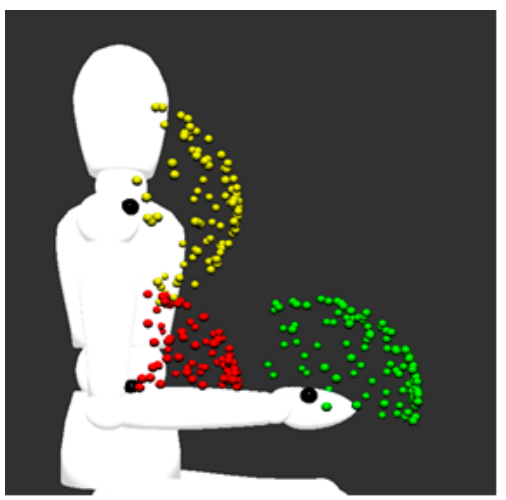

(e)

Figure 3. Part of touchers' hand positions when evaluator clicked a button to stop the hand. (a-c) show each body part data from front view and (d,e) show all body part data from both side views. Plotted data are relative to positions of 3D model's shoulder, elbow, and hands for visualization. Thus, actual postures of participants are different.

\subsection{Data Analysis Results}

4.1.1. Parts, Angle, and Gender Combination Effects

First, we analyzed the collected data from three factors: parts (shoulder, elbow and hand), angle (left and right), and gender combination (same or different), as shown in Tables 1 and 2. The reason why we use a gender combination factor is that a past study might suggest that pre-touch reaction distance increases with the opposite gender, although they did not provide strict evidence [16]. We conducted a three-factor mixed ANOVA test and only identified a significant main effect in the part factor $(\mathrm{F}(2,60)=13.905, p<0.001$, partial $\eta 2=0.317$ ). Multiple comparisons with the Bonferroni method revealed a significant 
difference for the distance factors: shoulder $>$ hand $(p=0.001)$ and elbow $>$ hand $(p=0.001)$. There was no significance between shoulder and elbow $(p=0.591)$. Thus, prediction 1 was supported.

Table 1. Average (S.E.) of minimum comfortable distance in centimeters by gender combinations and body parts.

\begin{tabular}{ccc}
\hline & \multicolumn{2}{c}{ Gender Combination } \\
\cline { 2 - 3 } & Same & Different \\
\hline Shoulder & $23.4(2.6)$ & $25.9(2.6)$ \\
Elbow & $23.6(2.5)$ & $24.1(2.5)$ \\
Hand & $21.0(2.3)$ & $21.3(2.3)$ \\
\hline
\end{tabular}

Table 2. Average (S.E.) of minimum comfortable distance in centimeters considering the approach angle for each body part.

\begin{tabular}{ccc}
\hline & \multicolumn{2}{c}{ Angle } \\
\cline { 2 - 3 } & Left & Right \\
\hline Shoulder & $25.7(2.0)$ & $23.6(1.8)$ \\
Elbow & $24.7(2.1)$ & $23.0(1,6)$ \\
Hand & $21.7(1.8)$ & $20.6(1.5)$ \\
\hline
\end{tabular}

Note that other factors did not show significant effects in all combinations, as shown in Table 3. Thus, in this data collection, only the body parts significantly affected the pre-touch reaction distance. In other words, the angle and the gender combinations between the touchers and the evaluators did not significantly affect the distance, similar to the past study [16].

Table 3. Three-factor ANOVA results $(p<0.05$ in bold).

\begin{tabular}{cccc}
\hline Factor & $p$-Value & Factor & $p$-Value \\
\hline Part $(\mathrm{P})$ & $<0.001$ & $\mathrm{P} \times \mathrm{A}$ & 0.623 \\
Angle $(\mathrm{A})$ & 0.083 & $\mathrm{P} \times \mathrm{G}$ & 0.200 \\
Gender combination $(\mathrm{G})$ & 0.749 & $\mathrm{~A} \times \mathrm{G}$ & 0.366 \\
& & $\mathrm{P} \times \mathrm{A} \times \mathrm{G}$ & 0.125 \\
\hline
\end{tabular}

\subsubsection{Additional Analysis: Speed Effect}

Next, we analyzed how the approach speed influenced the data collection, given that the interaction movement differed from person to person. We investigated this effect by investigating the relationship between the speed of the toucher's hand and the minimum comfortable distance and obtained a weak positive correlation $(\mathrm{r}=0.342, p<0.001)$. A faster movement might have caused more nervousness in the evaluator who reacted more quickly. In our setup, the defined pre-touch situation showed a weak positive correlation for the obtained minimum comfortable distance.

\subsubsection{Additional Analysis: Acclimation Effect}

Finally, we analyzed whether the minimum comfortable distance changed during data collection (Table 4). Since the participants were involved in many touch interactions, we wanted to know whether their perception influenced the measured distance. For this purpose, we investigated the acclimation factor by separating the data set into two classesfirst half and last half-and used the part factor because we found significant differences in the above analysis. Thus, we again conducted a two-factor (part and acclimation) ANOVA test and identified significant main effects in the part factor $(F(2,62)=14.216$, $p<0.001$, partial $\left.\eta^{2}=0.314\right)$ and the acclimation factor $(\mathrm{F}(1,31)=18.741, p<0.001$, partial 
$\left.\eta^{2}=0.377\right)$. We did not find any significance in the interaction effect $(F(2,62)=0.591$, $p=0.557$, partial $\eta^{2}=0.019$ ). Multiple comparisons with the Bonferroni method revealed a significant difference for the distance factors: shoulder $>$ hand $(p<0.001)$ and elbow $>$ hand $(p<0.001)$. There was no significance between the shoulder and elbow $(p=0.653)$.

Table 4. Average (S.E.) minimum comfortable distance in centimeters, considering acclimation.

\begin{tabular}{ccc}
\hline & \multicolumn{2}{c}{ Acclimation } \\
\cline { 2 - 3 } & First Half & Last Half \\
\hline Shoulder & $23.9(1.7)$ & $25.0(1.9)$ \\
Elbow & $22.9(1.5)$ & $24.5(1.8)$ \\
Hand & $20.8(1.7)$ & $21.4(1.6)$ \\
\hline
\end{tabular}

Thus, analysis of the part factor showed the same phenomenon as in the already supported prediction 1. Unlike a past study [16], our data collection showed that acclimation significantly affected the minimum comfortable distance, but this difference is about $1 \mathrm{~cm}$ on average.

\subsubsection{Summary}

Based on analysis for the pre-touch reaction distance around the upper body parts, we confirmed that the minimum comfortable distance around the hands is smaller than the minimum comfortable distance around the shoulders and elbows. Moreover, the results showed that gender and angle factors did not show a significant effect. These results exhibited a similar phenomenon with a past study about pre-touch reaction distance around the face [16]. The parts factor, only for the distance around the hands, showed a significant difference in the shoulder and elbow distances. The movement speed showed a weak impact in the minimum comfortable distance, and the acclimation effect also showed a significant difference.

\subsection{Implementation}

The next step is to implement the obtained pre-touch reaction distances on a robotic agent. We used an android robot with a masculine appearance [49] and an OptiTrack system that resembles our data collection to capture the body parts' positions in real-time. The robot reacted to the interaction when the hand approached the threshold distance using the markers' position information, based on the data collection results.

In the implementation of the pre-touch reactions for the robot, we prepared three boundaries for the shoulder, elbow, and hand and another for the face, based on a past study [16], to achieve pre-touch reaction behaviors for typical upper body parts. The boundary thresholds are defined as $\mathrm{T}_{\text {face }}, \mathrm{T}_{\text {shoulder }}, \mathrm{T}_{\text {elbow }}$, and $\mathrm{T}_{\text {hand }}$ from the positions of each body part of the robot, i.e., $\mathrm{P}_{\text {Rface }}, \mathrm{P}_{\text {Rshoulder }}, \mathrm{P}_{\text {Relbow }}$, and $\mathrm{P}_{\text {Rhand }}$. The distances between the participant's hand $\left(\mathrm{P}_{\text {Phand }}\right)$ and each body part of the robot were calculated by the Optitrack system's outputs $\left(\mathrm{D}_{\text {face }}, \mathrm{D}_{\text {shoulder }}, \mathrm{D}_{\text {elbow }}\right.$, and $\left.\mathrm{D}_{\text {hand }}\right)$. When this distance was equal to or less than a threshold (e.g., $\mathrm{D}_{\text {elbow }}<=\mathrm{T}_{\text {elbow }}$ ), the robot reacted and looked at the person, using as a reference the marker on the participant's head. We implemented this looking behavior because, in previous works, this reaction was adequate to obtain a more accurate distance [16]. In this implementation, we simply used the average values as the pre-touch reaction distance threshold for defining the model based on these analyses (Figure 4, shoulder $=24.8 \mathrm{~cm}$, elbow $=24.1 \mathrm{~cm}$, hand $=21.5 \mathrm{~cm}$ ). Note that we can change the system's thresholds by considering the angle and gender of interacting partners. 


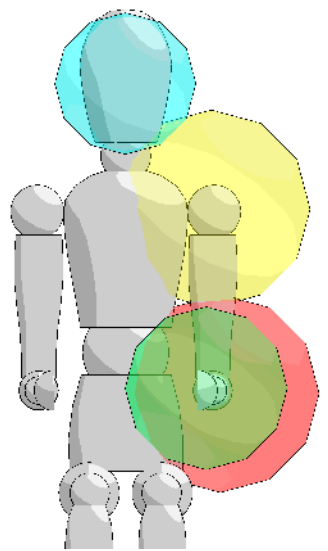

(a)

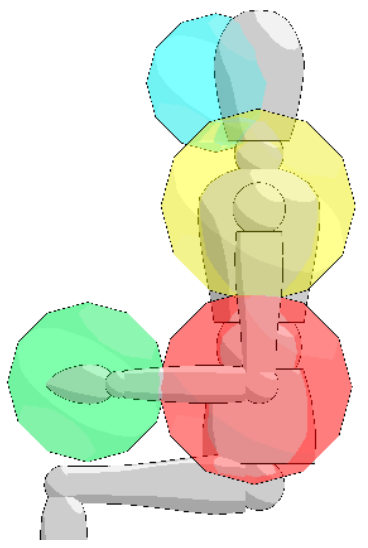

(b)

Figure 4. Visualizations of pre-touch reaction distance in proposed condition. (a) show the frontal view of the boundaries and (b) shows the lateral view of the boundaries. For readability, figure only shows boundaries for left body part (blue: Tface, yellow: Tshoulder, red: Telbow, green: Thand).

Finally, we tested our developed system. The touchers wore a marker on their right hand (fingers) and their head, as shown in Figure 5a, and were standing approximately $1.0 \mathrm{~m}$ from the robot's position, as shown in Figure $5 \mathrm{~b}$. Then, the touchers slowly moved their hands while approaching the robot's body part to check the robot's reaction. We designed the robot to react by looking at the touchers' face and confirmed that it autonomously reacted to the pre-touch behaviors (Figure 6) by testing with multiple different touchers.

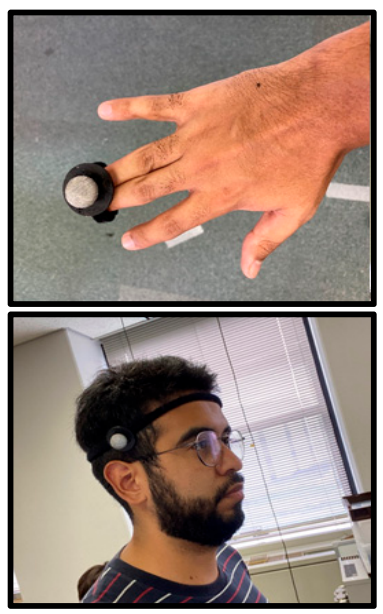

(a)

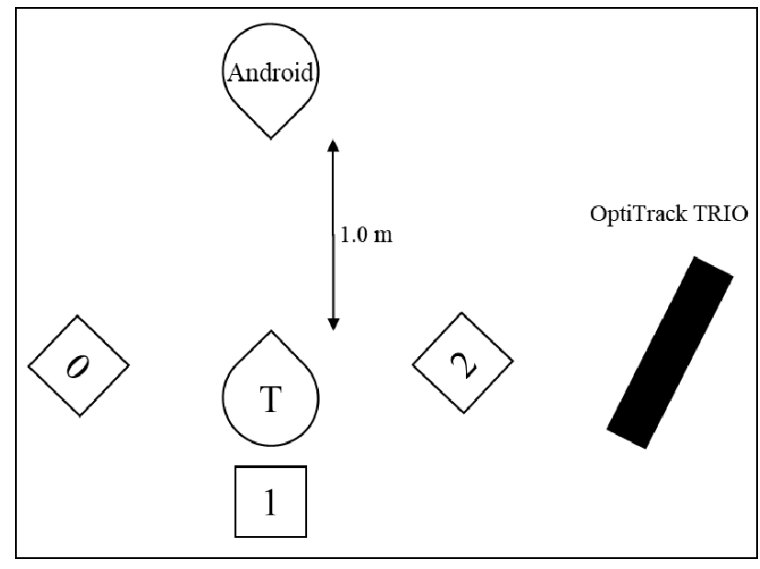

(b)

Figure 5. Implementation setup: (a) markers' positions on the participant: hand for computing distance and head as reference for android's reaction; (b) implementation setup using an OptiTrack system and three approaching angles: front, left, and right. 


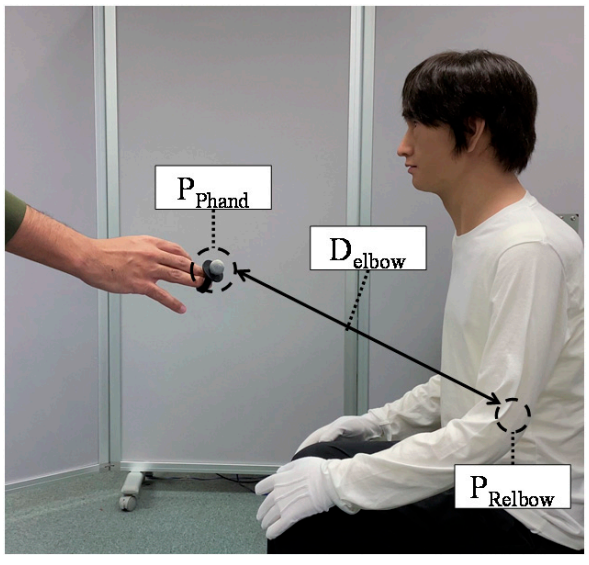

(a)

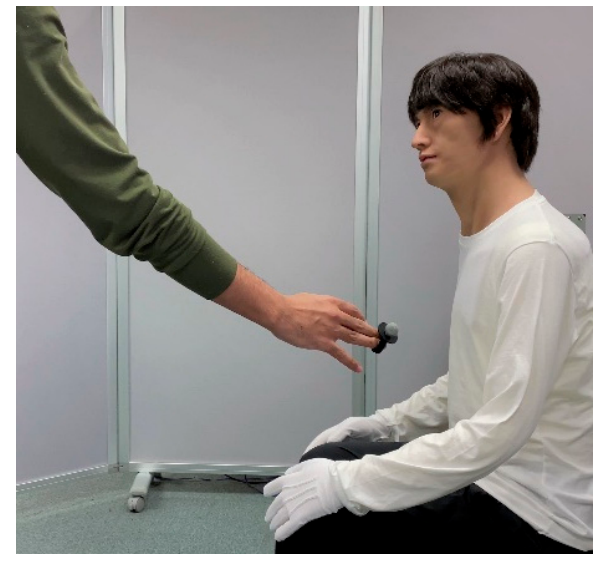

(b)

Figure 6. Reaction to potential touch to elbow implementing obtained model: (a) method used for detecting when the android should react considering the distance between the participant's hand and the android's elbow; (b) android pre-touch reaction.

\section{Discussion}

\subsection{Implications}

This study investigated and modeled the pre-touch reaction distance around socially touchable upper body parts, based on human-human pre-touch interaction observations. Even if we conducted our research with limited participants (e.g., number, age, and culture), our analyzed data provide useful knowledge for researchers interested in (pre-) touch interaction in both human-human and human-robot interactions.

In human science literature, females are more receptive to being touched or to engage in close-distance interaction than males. They also are more willing to accept a same-gender touch than an opposite-gender touch [50-52]. Even though the data collection analysis did not show significant differences, the gathered data may be useful to study gender effects of pre-touch distance around body parts.

Cultural differences are another possible factor that may have influenced the data collection results for pre-touch reaction distance. In fact, touch interaction is quite different due to cultural effects $[53,54]$ and situations $[55,56]$. In this study, only Japanese participants joined the data collection in a laboratory experiment setting. However, our findings might encourage comparisons of cultural differences in the context of pre-touch interactions.

In summary, our studies still identified a more complex phenomenon than past studies. These results suggest that robots might require a different strategy in (pre-) touch interaction situations due to the appearances and the genders of the interacting people.

\subsection{Implementation of Whole-Upper-Body Pre-Touch Reaction Distance}

This study implemented a pre-touch reaction distance threshold for face, shoulder, elbow, and hand based on past knowledge and observations. However, these distance thresholds are based on specific body parts. How should we deal with the pre-touch reaction distance between the above parts or others, such as the chest and stomach?

One possible solution is to interpolate linearly between body parts, enabling a robot to calculate the whole arm's pre-touch reaction distance. We may need to consider the sensitivity of other body parts, because a past study reported that touching such sensitive body parts is unacceptable except by a familiar person [18]. Investigating the relationship between such sensitivity and pre-touch reaction distance is an interesting future work.

The results obtained in this paper can be a starting point for modeling a pre-touch behavior in human-robot interaction. In this case, the reaction of the android is quite simple and only shows an "awareness" of a touch interaction by looking at the participant, but, for future work, it would be interesting to consider more complex reactions that depend on several factors, such as gender, age, and the context of the interaction. As for 
implementation with other kinds of robots, even if is not human-like, social robots should have human-like behavior when interacting with people, because people assume socially acceptable human behavior and expect a reaction accordingly.

Another interesting future work could be the inclusion of tactile data in the studied body parts. It would be helpful for finding a relationship between the touch interaction and the pre-touch reaction distance, comparing soft and hard touch with different beforetouch motion patterns (for example, pre-touch aggressive movement) and the effect in the conveyed emotions.

\subsection{Limitations}

Since this study has several limitations, we need to carefully contemplate our results. Such participant characteristics as personality, culture, and age affect the pre-touch reaction distance. In addition, we could not investigate the pre-touch reaction distance around the lower body due to difficulties observing human-human touch interaction. Although building whole-body pre-touch reaction distance knowledge is critical, such data collection is complicated by several issues, including ethical and privacy problems. Even if we used robots or virtual agents as touchers instead of human participants, data collection is problematic. Additionally, there are differences between real life and Virtual Reality (VR) environments regarding proxemics, where people prefer closer interaction distances with a physical robot than a virtual one in VR, suggesting that is important to evaluate the perception of the robot before the analysis of the interaction itself $[46,57]$.

In addition, since our model is based on participants who were essentially strangers, our study did not consider how it might change with people who already share some kind of relationship. Investigating these differences in human-human pre-touch interaction will be interesting for evaluating the same effect in human-robot interaction in the future.

\section{Conclusions}

This study focused on a before-touch situation in human-human touch interactions to define a pre-touch reaction distance and implement it using a human-like male android. We reported the modeling of pre-touch reaction distance around a touchable upper body part, i.e., shoulders, elbows, and hands, from human-human interaction observations considering gender and the side approach and speed of the touch interaction. The pretouch reaction distances of the shoulder and elbow had a significantly higher value, while the pre-touch reaction distance for the hand had a significantly lower value. Moreover, our analysis showed that speed and acclimation significantly affected the distance, although gender and approach sides did not.

We also implemented a pre-touch reaction distance model in human-robot pre-touch interaction. We used an android robot with a human-like masculine appearance that reacted based on the defined reaction distances obtained in the human-human interaction analysis. Based on the robot's appearance, knowledge about upper-body pre-touch reaction distance based on human-human touch interaction and the differences in its effectiveness will contribute to gathering pre-touch proxemics knowledge for social robots that physically interact with people.

Author Contributions: Conceptualization, D.A.C.M., H.S., and M.S.; methodology, D.A.C.M. and M.S.; software, D.A.C.M.; validation, D.A.C.M., H.S., and M.S.; formal analysis, D.A.C.M., H.S., and M.S.; investigation, D.A.C.M., H.S., and M.S.; resources, D.A.C.M., H.S., and M.S.; data curation, D.A.C.M., H.S., and M.S.; writing-original draft preparation, D.A.C.M., H.S., and M.S.; writingreview and editing, D.A.C.M., H.S., and M.S.; visualization, D.A.C.M.; supervision, H.S., H.I., and M.S.; project administration, H.I. and M.S.; funding acquisition, H.I. and M.S. All authors have read and agreed to the published version of the manuscript.

Funding: This work was supported, in part, by JST CREST Grant Number JPMJCR18A1, Japan, and JST ERATO Ishiguro Symbiotic Human Robot Interaction Project (Grant Number: JPMJER1401). 
Institutional Review Board Statement: The study was approved by the ethics committee at the Advanced Telecommunication Research Institute (ATR) (20-501-4).

Informed Consent Statement: Informed consent was obtained from all subjects involved in the study.

Data Availability Statement: The data presented in this study can be obtained on request from the corresponding author.

Acknowledgments: We thank Sayuri Yamauchi for her help during the execution of our experiments.

Conflicts of Interest: The authors declare no conflict of interest. The funders had no role in the design of the study; in the collection, analyses, or interpretation of data; in the writing of the manuscript, or in the decision to publish the results.

\section{References}

1. Grewen, K.M.; Anderson, B.J.; Girdler, S.S.; Light, K.C. Warm Partner Contact Is Related to Lower Cardiovascular Reactivity. Behav. Med. 2003, 29, 123-130. [CrossRef]

2. Cohen, S.; Janicki-Deverts, D.; Turner, R.B.; Doyle, W.J. Data from: Does hugging provide stress-buffering social support? A study of susceptibility to upper respiratory infection and illness. Psychol. Sci. 2015, 26, 135-147. [CrossRef]

3. Jakubiak, B.K.; Feeney, B.C. Keep in touch: The effects of imagined touch support on stress and exploration. J. Exp. Soc. Psychol. 2016, 65, 59-67. [CrossRef]

4. Gallace, A.; Spence, C. The science of interpersonal touch: An overview. Neurosci. Biobehav. Rev. 2010, 34, 246-259. [CrossRef] [PubMed]

5. Light, K.C.; Grewen, K.M.; Amico, J.A. More frequent partner hugs and higher oxytocin levels are linked to lower blood pressure and heart rate in premenopausal women. Biol. Psychol. 2005, 69, 5-21. [CrossRef] [PubMed]

6. Field, T. Touch for socioemotional and physical well-being: A review. Dev. Rev. 2010, 30, 367-383. [CrossRef]

7. Yu, R.; Hui, E.; Lee, J.; Poon, D.; Ng, A.; Sit, K.; Ip, K.; Yeung, F.; Wong, M.; Shibata, T.; et al. Use of a Therapeutic, Socially Assistive Pet Robot (PARO) in Improving Mood and Stimulating Social Interaction and Communication for People with Dementia: Study Protocol for a Randomized Controlled Trial. JMIR Res. Protoc. 2015, 4, e45. [CrossRef] [PubMed]

8. Sumioka, H.; Nakae, A.; Kanai, R.; Ishiguro, H. Huggable communication medium decreases cortisol levels. Sci. Rep. 2013, 3, 3034. [CrossRef] [PubMed]

9. Shiomi, M.; Nakagawa, K.; Shinozawa, K.; Matsumura, R.; Ishiguro, H.; Hagita, N. Does A Robot's Touch Encourage Human Effort? Int. J. Soc. Robot. 2016, 9, 5-15. [CrossRef]

10. Shiomi, M.; Nakata, A.; Kanbara, M.; Hagita, N. A hug from a robot encourages prosocial behavior. In Proceedings of the 2017 26th IEEE International Symposium on Robot and Human Interactive Communication (RO-MAN), Lisbon, Portugal, 28 August-1 September 2017; pp. 418-423. [CrossRef]

11. Bevan, C.; Fraser, D.S. Shaking Hands and Cooperation in Tele-present Human-Robot Negotiation. In Proceedings of the Tenth Annual ACM/IEEE International Conference on Human-Robot Interaction, Portland, OR, USA, 2-5 March 2015; pp. $247-254$.

12. Shiomi, M.; Nakata, A.; Kanbara, M.; Hagita, N. A Robot that Encourages Self-disclosure by Hug. In Proceedings of the Social Robotics: 9th International Conference, ICSR 2017, Tsukuba, Japan, 22-24 November 2017; Kheddar, A., Yoshida, E., Ge, S.S., Eds.; Springer International Publishing: Cham, Switzerland, 2017; pp. 324-333.

13. Shiomi, M.; Hagita, N. Do Audio-Visual Stimuli Change Hug Impressions? In Proceedings of the Social Robotics: 9th International Conference, ICSR 2017, Tsukuba, Japan, 22-24 November 2017; Kheddar, A., Yoshida, E., Ge, S.S., Eds.; Springer International Publishing: Cham, Switzerland, 2017; pp. 345-354.

14. Chen, T.L.; King, C.-H.A.; Thomaz, A.L.; Kemp, C.C. An Investigation of Responses to Robot-Initiated Touch in a Nursing Context. Int. J. Soc. Robot. 2014, 6, 141-161. [CrossRef]

15. Fukuda, H.; Shiomi, M.; Nakagawa, K.; Ueda, K. 'Midas touch' in human-robot interaction: Evidence from event-related potentials during the ultimatum game. In Proceedings of the 20127 th ACM/IEEE International Conference on Human-Robot Interaction (HRI), Boston, MA, USA, 5-8 March 2012; pp. 131-132.

16. Shiomi, M.; Shatani, K.; Minato, T.; Ishiguro, H. How should a Robot React before People's Touch?: Modeling a Pre-Touch Reaction Distance for a Robot's Face. IEEE Robot. Autom. Lett. 2018, 3, 3773-3780. [CrossRef]

17. Sato, A.; Kimoto, M.; Iio, T.; Shimohara, K.; Shiomi, M. Preliminary Investigation of Pre-Touch Reaction Distances toward Virtual Agents. In Proceedings of the 7th International Conference on Human-Agent Interaction, Kyoto, Japan, 6-10 October 2019; Association for Computing Machinery (ACM): New York, NY, USA, 2019; pp. 292-293.

18. Suvilehto, J.T.; Glerean, E.; Dunbar, R.I.M.; Hari, R.; Nummenmaa, L. Topography of social touching depends on emotional bonds between humans. Proc. Natl. Acad. Sci. USA 2015, 112, 13811-13816. [CrossRef] [PubMed]

19. Hall, E.T. The Hidden Dimension; Doubleday: Garden City, NY, USA, 1966.

20. Kirby, R.; Simmons, R.; Forlizzi, J. COMPANION: A Constraint-Optimizing Method for Person-Acceptable Navigation. In Proceedings of the RO-MAN 2009-The 18th IEEE International Symposium on Robot and Human Interactive Communication, Toyama, Japan, 27 September-2 October 2009; pp. 607-612. 
21. Luber, M.; Spinello, L.; Silva, J.; Arras, K.O. Socially-aware robot navigation: A learning approach. In Proceedings of the 2012 IEEE/RSJ International Conference on Intelligent Robots and Systems, Algarve, Portugal, 7-12 October 2012; pp. 902-907.

22. Svenstrup, M.; Bak, T.; Andersen, H.J. Trajectory planning for robots in dynamic human environments. In Proceedings of the 2010 IEEE/RSJ International Conference on Intelligent Robots and Systems, Taipei, Taiwan, 18-22 October 2010; pp. $4293-4298$.

23. Gómez, J.V.; Mavridis, N.; Garrido, S. Social path planning: Generic human-robot interaction framework for robotic navigation tasks. In Proceedings of the 2nd International Workshop on Cognitive Robotics Systems: Replicating Human Actions and Activities, Tokyo, Japan, 3 November 2013.

24. Satake, S.; Kanda, T.; Glas, D.F.; Imai, M.; Ishiguro, H.; Hagita, N. A Robot that Approaches Pedestrians. IEEE Trans. Robot. 2012, 29, 508-524. [CrossRef]

25. Huang, C.-M.; Iio, T.; Satake, S.; Kanda, T. Modeling and Controlling Friendliness for An Interactive Museum Robot. In Proceedings of the Robotics: Science and Systems X, Berkely, CA, USA, 12-16 July 2014; Robotics: Science and Systems Foundation, University of California: Berkely, CA, USA, 2014.

26. Takayama, L.; Pantofaru, C. Influences on proxemic behaviors in human-robot interaction. In Proceedings of the 2009 IEEE/RSJ International Conference on Intelligent Robots and Systems, Hyatt Regency, St. Louis, MO, USA, 11-15 October 2009.

27. Mumm, J.; Mutlu, B. Human-robot proxemics: Physical and psychological distancing in human-robot interaction. In Proceedings of the 6th International Conference on Human-Robot Interaction, Lausanne, Switzerland, 6-9 March 2011; pp. 331-338.

28. Rossi, S.; Staffa, M.; Bove, L.; Capasso, R.; Ercolano, G. User's Personality and Activity Influence on HRI Comfortable Distances. In Proceedings of the Transactions on Petri Nets and Other Models of Concurrency XV; Springer Science and Business Media LLC: Berlin, Germany, 2017; pp. 167-177.

29. Hiroi, Y.; Ito, A. Influence of the Size Factor of a Mobile Robot Moving toward a Human on Subjective Acceptable Distance. In Mobile Robots_Current Trends; Zoran Gacovski, Z., Ed.; InTechOpen Europe: Rikeja, Croatia, 2011. [CrossRef]

30. Kim, Y.; Kwak, S.S.; Kim, M.-S. Am I acceptable to you? Effect of a robot's verbal language forms on people's social distance from robots. Comput. Hum. Behav. 2013, 29, 1091-1101. [CrossRef]

31. Obaid, M.; Sandoval, E.B.; Złotowski, J.; Moltchanova, E.; Basedow, C.A.; Bartneck, C. Stop! That is close enough. How body postures influence human-robot proximity. In Proceedings of the 2016 25th IEEE International Symposium on Robot and Human Interactive Communication (RO-MAN), New York, NY, USA, 26-31 August 2016; pp. 354-361.

32. Abe, K.; Hamada, Y.; Nagai, T.; Shiomi, M.; Omori, T. Estimation of child personality for child-robot interaction. In Proceedings of the 2017 26th IEEE International Symposium on Robot and Human Interactive Communication (RO-MAN), Lisbon, Portugal, 28-31 August 2017; pp. 910-915. [CrossRef]

33. Anzalone, S.M.; Varni, G.; Ivaldi, S.; Chetouani, M. Automated Prediction of Extraversion During Human-Humanoid Interaction. Int. J. Soc. Robot. 2017, 9, 385-399. [CrossRef]

34. Shiomi, M.; Hagita, N. Audio-Visual Stimuli Change Not Only Robot's Hug Impressions but Also Its Stress-Buffering Effects. Int. J. Soc. Robot. 2021, 13, 469-476. [CrossRef]

35. Chen, T.L.; Bhattacharjee, T.; Beer, J.; Ting, L.H.; Hackney, M.E.; Rogers, W.A.; Kemp, C.C. Older adults' acceptance of a robot for partner dance-based exercise. PLoS ONE 2017, 12, e0182736. [CrossRef] [PubMed]

36. Chen, T.L.; Bhattacharjee, T.; McKay, J.L.; Borinski, J.E.; Hackney, M.E.; Ting, L.H.; Kemp, C.C. Evaluation by Expert Dancers of a Robot That Performs Partnered Stepping via Haptic Interaction. PLoS ONE 2015, 10, e0125179. [CrossRef]

37. Kosuge, K.; Hayashi, T.; Hirata, Y.; Tobiyama, R. Dance partner robot-ms dancer. In Proceedings of the 2003 IEEE/RSJ International Conference on Intelligent Robots and Systems, Las Vegas, NV, USA, 27-31 October 2003; pp. 3459-3464.

38. Hirano, T.; Shiomi, M.; Iio, T.; Kimoto, M.; Tanev, I.; Shimohara, K.; Hagita, N. How Do Communication Cues Change Impressions of Human-Robot Touch Interaction? Int. J. Soc. Robot. 2017, 10, 21-31. [CrossRef]

39. Suvilehto, J.T.; Nummenmaa, L.; Harada, T.; Dunbar, R.I.M.; Hari, R.; Turner, R.; Sadato, N.; Kitada, R. Cross-cultural similarity in relationship-specific social touching. Proc. R. Soc. B Boil. Sci. 2019, 286, 20190467. [CrossRef]

40. Hertenstein, M.J.; Keltner, D.; App, B.; Bulleit, B.A.; Jaskolka, A.R. Touch communicates distinct emotions. Emotion 2006, 6, 528-533. [CrossRef] [PubMed]

41. Alenljung, B.; Andreasson, R.; Lowe, R.; Billing, E.; Lindblom, J. Conveying Emotions by Touch to the Nao Robot: A User Experience Perspective. Multimod. Technol. Interact. 2018, 2, 82. [CrossRef]

42. Zheng, X.; Shiomi, M.; Minato, T.; Ishiguro, H. What Kinds of Robot's Touch Will Match Expressed Emotions? IEEE Robot. Autom. Lett. 2019, 5, 127-134. [CrossRef]

43. Prasad, V.; Stock-Homburg, R.; Peters, J. Human-Robot Handshaking: A Review. Int. J. Soc. Robot. 2021, 1-17. [CrossRef]

44. Stock-Homburg, R.; Peters, J.; Schneider, K.; Prasad, V.; Nukovic, L.; Darmstadt, D.R.S.-H.T.U. Evaluation of the Handshake Turing Test for anthropomorphic Robots. In Proceedings of the Companion of the $2020 \mathrm{ACM} / \mathrm{IEEE}$ International Conference on Human-Robot Interaction, Cambridge, UK, 23-26 March 2020.

45. Lehmann, H.; Rojik, A.; Hoffmann, M. Should a small robot have a small personal space? Investigating personal spatial zones and proxemic behavior in human-robot interaction. In Proceedings of the CognitIve RobotiCs for intEraction (CIRCE) Workshop at IEEE International Conference on Robot and Human Interactive Communication (RO-MAN), Naples, Italy, 31 August-4 September 2020. 
46. Li, R.; Van Almkerk, M.; Van Waveren, S.; Carter, E.J.; Leite, I. Comparing Human-Robot Proxemics Between Virtual Reality and the Real World. In Proceedings of the 2019 14th ACM/IEEE International Conference on Human-Robot Interaction (HRI), Daegu, Korea, 11-14 March 2019; pp. 431-439.

47. Amaoka, T.; Laga, H.; Saito, S.; Nakajima, M. Personal Space Modeling for Human-Computer Interaction. In Proceedings of the Transactions on Petri Nets and Other Models of Concurrency XV; Springer Science and Business Media LLC: Berlin, Germany, 2009; pp. $60-72$.

48. Svenstrup, M.; Tranberg, S.; Andersen, H.J.; Bak, T. Pose estimation and adaptive robot behaviour for human-robot interaction. In Proceedings of the 2009 IEEE International Conference on Robotics and Automation, Kobe, Japan, 12-17 May 2009; pp. 3571-3576.

49. Shiomi, M.; Sumioka, H.; Sakai, K.; Funayama, T.; Minato, T. SŌTO: An Android Platform with a Masculine Appearance for Social Touch Interaction. In Proceedings of the Companion of the 2020 ACM/IEEE International Conference on Human-Robot Interaction, Cambridge, UK, 23-26 March 2020; pp. 447-449.

50. Baldassare, M.; Feller, S. Cultural Variations in Personal Space: Theory, Methods, and Evidence. Ethos 1975, 3, 481-503. [CrossRef]

51. Heshka, S.; Nelson, Y. Interpersonal speaking distance as a function of age, sex, and relationship. Sociometry 1972, 35, 491-498. [CrossRef]

52. Knapp, M.L.; Hall, J.A.; Horgan, T.G. Nonverbal Communication in Human Interaction; Cengage Learning: Boston, MA, USA, 2013.

53. McDaniel, E.; Andersen, P.A. International Patterns of Interpersonal Tactile Communication: A Field Study. J. Nonverbal Behav. 1998, 22, 59-75. [CrossRef]

54. DiBiase, R.; Gunnoe, J. Gender and Culture Differences in Touching Behavior. J. Soc. Psychol. 2004, 144, 49-62. [CrossRef]

55. Hall, J.A. Touch, status, and gender at professional meetings. J. Nonverbal Behav. 1996, 20, 23-44. [CrossRef]

56. Willis, F.N.; Briggs, L.F. Relationship and touch in public settings. J. Nonverbal Behav. 1992, 16, 55-63. [CrossRef]

57. Cuello Mejía, D.A.; Saito, A.; Kimoto, M.; Iio, T.; Shimohara, K.; Sumioka, H.; Ishiguro, H.; Shiomi, M. Modeling of Pre-Touch Reaction Distance for Faces in a Virtual Environment. J. Inf. Process. (JIP) 2021, 29. to be published. 